\title{
Spotlights on new publications
}

\author{
Sherif M Abaza
}

Medical Parasitology Department, Faculty of Medicine, Suez Canal Uinversity, Ismailia, Egypt

Corresponding Author: Tel.: 01005243428, E-mail: smabaza@hotmail.com

Received: 2 February, 2019, Accepted: 20 February, 2019.

Print ISSN: 1687- 7942, Online ISSN: 2090 -2646, Vol. 12, No. 1, April, 2019

\section{New drug targets IX}

Malignant malaria: Although proteases constitute only $20 \%$ of $P$. falciparum genome, they are involved in several functions either for parasite growth, e.g. host hemoglobin degradation and exporting protein essential for parasite genome, or evading host immune response, e.g. processing surface antigen. Trophozoite stage expresses two major cysteine proteases (CPs); falcipain-2 (FP2) and falcipain-3 (FP3). Both CPs ( $\sim 50$ $\mathrm{kDa}$ ) are formed of pro- and mature domains that interact together via 'hot-spot' interactions to maintain the structural integrity of enzyme in zymogen state (i.e. inactive precursor of the enzyme). Meanwhile, zymogen requires a biochemical change, which usually occurs in Golgi bodies, to reveal its active site. After synthesis, they are transported through the endoplasmic reticulum/ Golgi network to an acidic environment (trophozoite food vacuole), where both CPs express the $\sim 27 \mathrm{kDa}$ active enzyme. On the other hand, expressions of FP2 and FP3 open wider posibilities for the use of several inhibitors such as E-64, leupeptin, vinyl sulfones, and falstatin in treatment of malignant malaria. Such inhibitors block the active sites of FP2 and FP3, while in the present compilation, Abhilasha Pant and his Indian colleagues presented the first trial to use allosteric inhibitors. Biochemically, allosteric control means regulation of an enzyme by binding an effector molecule at a site other than its active site. The site to which the effector binds; allosteric site; allows effectors to change enzyme dynamics. Effectors increasing the enzyme activity are called allosteric activators, and vice versa. Use of allosteric inhibitors in treatment of human diseases such as neurodegenerative diseases, and Kaposi's sarcoma-associated herpesvirus, encouraged the investigators to search for novel allosteric falcipain inhibitor. Besides, active site inhibitors, being highly conserved, are confronted by high drug pressure, and more susceptibility to gene mutations commonly linked with resistance to several anti-malarial drugs. On the other hand, allosteric inhibitors are more selective and potent. Moreover, gene mutation never occurs in presence of high drug pressure because these inhibitors don't alter the active site.

The investigators hypothesized that azapeptides, as new generation of allosteric inhibitors, could be novel anti-malarial drugs against resistant strains of $P$. falciparum. Additionally, it was reported that histidine kinase of $H$. pylori requires H94 residue to sense the surrounding $\mathrm{pH}$ for subsequent auto-phosphorylation. Therefore, the second objective of the present compilation was to identify the individual histidine residue in both CPs responsible for sensing the acidic $\mathrm{pH}$ and initiation of auto-processing and proteolytic maturation. Out of six synthesized azapeptides, only two (NA-1 and NA-3) arrested P. falciparum in vitro growth on a dose-dependent manner through blocking the auto-processing of falcipains. In addition, both compounds have no activity against human peripheral blood mononuclear cells, and NA-3 showed five-fold better activity compared to the active site inhibitor (E64). There was a significant reduction in parasitemia at trophozoite stage with accumulation of un-degraded hemoglobin in its food vacuole, suggesting inhibition of falcipain auto-processing as well as proteolytic maturation. Also, it was observed that NA-3-treated parasites showed distorted morphology that remained undeveloped for 48 hours suggesting growth arrest. However, only partial inhibition was occasionally observed, and the investigators attributed this to the occurrence of falcipains processing in a cellular compartment with high lipid cell wall content. For the secondary objective, the investigators identified that histidine at 205 position (H205) may be the individual histidine residue for sensing the acidic $\mathrm{pH}$ and initiation of falcipains auto-processing and proteolytic maturation.

The investigators concluded that allosteric inhibitors are able to bind at the interface of the promature domains, block the 'hot-spot' interactions and interfere with falcipains auto-processing. Finally, the investigators recommended future studies to synthesize more azapeptides compounds with high lipophilicity to access such cellular compartments with higher potency. Compiled from "Allosteric site inhibitor disrupting auto-processing of malarial cysteine proteases." Sci Rep 2018 Nov 1; 8(1): 16193.

Schistosomiasis: It is well known that praziquantel (PZQ), the sole WHO-licensed chemotherapy for 
schistosomiasis in the past decades, increases schistosomes tegument's permeability to calcium ions, leading to spastic muscle contraction, and death of adult worms. Other mechanisms of action were postulated such as oviposition disturbances, and interference with adenosine uptake in worms that are unable to synthesize purines. Praziquantel is administered as a racemate, with only its ( $\mathrm{R}$ )-enantiomer as the biologically active component. A racemic mixture is characterized by having equal left- and right-handed enantiomers of a chiral molecule, while an enantiomer is one of two stereoisomers (mirror images of each other that are non-superposeable), i.e. they are not identical. This meaning can be simplified by imagining that left and right hands are the same except for being reversed along one axis. In most instances, only one of a drug's enantiomers is responsible for its chemotherapeutic effects. On the other hand, chemical synthesis of enantiomeric compounds such as PZQ (i.e. in a racemic mixture) means its inevitable separation into its enantiomeric components in the absence of enantiomeric environment (precursor, chiral catalyst, or kinetic resolution). Meanwhile, G-protein coupled receptors (GPCRs) were found to have major roles in flat worms' movement, sexual maturation and eggs production. Previous studies showed that union of serotonin (5-HT) with GPCRs results in enhancement of neuromuscular functions via cAMP coupling to regulate parasite movement. To postulate the target molecule(s) of PZQ, a recent study was conducted by an American group of scientists and showed that PZQ (R)enantiomer acts as a GPCR ligand. The investigators also showed the pharmacological action(s) of schistosome serotonergic GPCR (Sm.5HTRL) in controlling adults movement. In addition, they found several heterocyclic alkaloids that regulate $S m .5 \mathrm{HTRL}$ activity, among them ergot alkaloids (ergot is a group of fungi of the genus Claviceps) such as tryptamine, aporphine and protoberberine. Because ergot alkaloids are used to regulate mammalian 5-HT receptors, Jonathan S. Marchant et al., investigated their role as Sm.5HTRL regulators. The objectives of the present compilation were to understand their pharmacological characteristic features that are involved in selectivity and potency for Sm.5HTRL, and to identify new target molecule(s) that can be used for development of novel $\operatorname{drug}(\mathrm{s})$.

To achieve their goals, they utilized cAMP luminescence assays to perform structure-activity studies to investigate 143 compounds that contain one of the ergot alkaloids (tryptamines, aporphines and protoberberines). Schistosome serotonin receptor (Sm.5HTRL) was expressed in culture cell lines, and the investigators observed, after addition of 5-HT, a dosedependent rapid increase in cAMP-luminescence only in cells expressing Sm.5HTRL. This was followed by testing the sensitivity and selectivity of this response by addition of the selected compounds instead of 5-HT. It is worth mentioning that structure modification of tryptamine derivatives (i.e. substitution of their active sites) agonist Sm.5HTRL activity. The investigators hypothesized that Sm.5HTRL ligands will stimulate or block the receptors used in schistosomules and adults movement. According to the results obtained from cAMP luminescence assays, the investigators identified several compounds belonging to substituted tryptamines as Sm.5HTRL agonists, while those that belong to aporphines and protoberberines were identified as Sm.5HTRL antagonists. Moreover, their functional active sites were characterized. This was followed by investigation of the efficacy of the identified compounds against in vitro cultured adult schistosomes.

Results showed that substituted tryptamines agonists and compounds sharing a benzylisoquinoline core, e.g. nuciferine (aporphines) and L-rotundine (protoberberines), stimulated worm movement, with decreased potency relative to results from cAMP luminescence assays; however, nuciferine was the most potent antagonist. The obtained results suggested that 1) serotonin receptor (5-HT), as essential regulator of neuromuscular activity, is recognized as an attractive target for anti-helminthic drug development; 2) both substituted tryptamines and natural products of benzylisoquinoline are considered serotonergic ligands, and 3) structure-activity profiling using cAMP luminescence assays is a good approach for screening compounds to search for suitable tolerable sites for serotonergic ligands. It was observed that substituted tryptamines that were tolerated in cAMP luminescence assays were also tolerated as serotonergic ligands when investigated against in vitro cultured adult schistosomes.

The investigators concluded that GPCRs are drug targets for development of novel anthelminthic chemotherapy, and recommended use of structureactivity profiling as an approach to investigate other schistosome GPCRs for future studies that aim to discover drug targets and develop novel drugs. Compiled from "Structure-activity profiling of alkaloid natural product pharmacophores against a Schistosoma serotonin receptor." Int J Parasitol Drugs Drug Resist 2018 Dec; 8(3): 550-558.

Filariasis: Filarial nematodes cause several diseases throughout all tropical and subtropical countries. Among them, lymphatic filariasis and onchocerciasis (river blindness) caused by W. bancrofti and B. malayi, and $O$. volvulus, respectively are associated with high morbidity. Massive drug programs are based on combinational administration of any two drugs among ivermectin (IVM), diethylcarbamazine (DEC), and albendazole (ALB) in treatment of lymphatic filariasis. While ivermectin is the sole chemotherapeutic agent in treatment of onchocerciasis. Besides, the long-living filarial worms, and the association of both diseases 
in some endemic areas necessitate urgently the development of novel drugs that affect new molecular targets in these filarial nematodes. Korean scientists (Tae-Woo Choi and his colleagues) proposed calcium binding protein (calumenin, CALU) as a novel drug target for filarial worms because its malfunction leads to reduced fertility as well as enhanced drug entry due its role in cuticle development. Meanwhile, molecular modeling showed significant distinct structures of nematode CALUs from the human counterpart. It was found that the gene (ID: 831) encodes biosynthesis of calcium-binding protein localized in the endoplasmic reticulum and involved in protein folding and sorting. This protein belongs to a CERC family with multiple proteins including reticulocalbin, ERC-55, and Cab45. Expression of this protein was reported in the pharynx, intestine, muscle, and hypodermis during development of the free-living nematode, $C$. elegans.

The results of a previous study conducted by the same group of investigators (Cho JH et al., 2009) revealed that CALU-mutant $C$. elegans showed reduced fertility with severe cuticle structural defects. In the present compilation, the objective is to search for an ideal novel compound with high affinity to bind specifically with nematode CALUs, not with human CALU. Besides, it should be a compound with no severe adverse reactions associated with dead worms, as well as with safe usage for both children and pregnant or breastfeeding women. On the other hand, advanced molecular evolution enabled the scientists to predict three-dimensional (3D) structures of proteins of interest, followed by ligand-binding studies and computational virtual screening, supplemented by FDA-approved drugs for new indications.

Because CALU has a crucial role in normal cuticle development where its malfunction increases drug entry, in addition to its sensitivity to kill adult worms, the investigators hypothesized that filarial nematodes CALU could serve as a novel drug target. To test this hypothesis, three $\mathrm{C}$. elegans strains were obtained, the wild type (N2) and two mutant strains for collagen and CALU biosynthesis. It is important to mention that normal cuticle development is crucial for normal growth of nematodes during larval molting to expand its body size. In this regard, cuticle collagen is a complex process, and CALU may have a role in its biosynthesis, which explains the addition of collagen-mutant strain in the study. C. elegans strains were cultivated without treatment and with ALB and IVM and the results revealed significant enhanced sensitivity to ALB and IVM in CALU-mutant strain compared to N2 strain. The hypersensitivity of CALU-mutant strain to ALB was observed in all drug concentrations tested, while with IVM, the only significant difference was obtained at one IVM concentration. On the other hand, no significance was detected between treated and untreated N2 strain. Interestingly, collagen-mutant strain, compared to N2 strain, showed similar results as CALU-mutant strain for ALB and IVM, even at the same significant IVM concentration. This was followed by amino acid sequences of human and filarial nematodes (C. elegans, B. malayi, and O. volvulus) CALUs to predict its 3D structure. It was found that nematode CALUs shared well-conserved structural features (91\% identity), but not with human CALU, suggesting that it could be strongly considered as a nematode-specific drug target.

The investigators extended their studies to screen the predicted 3D models of nematodes CALUs (structure-based virtual screening), using molecular docking. A library of 1700 commercially available FDA-approved drugs was screened to select the best compounds that specifically bind to nematode CALUs, but not to human CALU. Fifty compounds were ranked, among them itraconazole (ICT), an inhibitor of sterol biosynthesis as well as anti-fungal agent, was selected as a potential drug to bind and specifically inhibit nematode CALUs. The investigators claimed that sterols have crucial role in several cellular functions, whereas both free-living and parasitic nematodes are unable to synthesize sterols de novo. To test ICT efficacy to target nematode CALUs, the investigators performed a survival assay using the same $C$. elegans strains previously used. CALU-mutant strain showed resistance to ICT in all concentrations except only at $160 u$ M ICT concentration at which it showed significant difference compared to N2 strain. On the other hand, significant results were obtained in both ICT-treated $\mathrm{N} 2$ (40 $\mu \mathrm{M}$ and above) and collagen-mutant strains (all drug concentrations). Accordingly, the investigators suggested that ITC selectively targets nematode CALUs and could be a novel drug for treatment of lymphatic filariasis and onchocerciasis. Compiled from "Novel findings of anti-filarial drug target and structurebased virtual screening for drug discovery." Int J Mol Sci 2018 Nov 13; 19(11): 5379.

Visceral leishmaniasis (VL): Several Leishmania species are incriminated in causing VL in wide range of mammals, including man, throughout the world, especially in endemic areas such as Brazil. Among them, $L$. donovani is the most prevalent in East Africa and India, whereas $L$. infantum predominates in Europe, North Africa, and Latin America. WHO placed VL as one of the major neglected tropical diseases because there is much discrepancy between the urgent need for safe and efficient drug and the number of both infected patients and individuals at risk, beside the complicated sequelae associated with improperly treated patients. In general, an important issue in development of a novel safe efficient drug is to search for a new component essential for parasite survival, but absent in its host. Specifically for Leishmania species because it inhabits one of the host's immune response components (macrophages), there was increased difficulty to find such modules for development of new drugs. It is well known that infected macrophages express hydrogen 
peroxide as one of the reactive oxidative species (ROS) against intracellular pathogen. In contrast to glutathione-based redox defense mechanism in mammals, blood flagellates possess trypanothione (TS2) that is reduced by trypanothione reductase (TR) to form $\mathrm{T}(\mathrm{SH}) 2$. Therefore, Leishmania parasites are able to couple $\mathrm{T}(\mathrm{SH}) 2$ with tryparedoxin/tryparedoxin peroxidase I to neutralize hydrogen peroxide produced by infected macrophages. On the other hand, as all trials to obtain TS2-mutant Leishmania strains failed, TR in Leishmania species, due to its role in detoxifying system against oxidative damage of the infected macrophages, is proposed as novel drug target. A group of investigators from Italy (Lorenzo Turcano, and his colleagues) hypothesized that compounds targeting TR, essential for parasite survival and absent in its human host, are new exclusive drugs for treatment of VL.

Nicotinamide adenine dinucleotide phosphate (NADP) is a cofactor used in several reactions that require NADPH as a reducing agent in synthesis of vital cellular compounds (e.g. lipids and nucleic acids). Meanwhile, NADPH is required to bind with TR to neutralize $\mathrm{H} 2 \mathrm{O} 2$ produced by Leishmania-infected macrophages. Several reports showed high similarity in TR crystal structure in trypanosomatids, as well as its binding domain with NADPH. Also, several publications used metals and metal-containing compounds to block the active binding site of NADPH with TR. In the present compilation, the investigators developed and validated a new luminescence-based high throughput screening (HTS) as a new method in which NADPH oxidation was coupled to a luminescence assay. Utilizing HTS with their in-house library containing 120,000 compounds allowed the investigators to identify new Leishmania TR inhibitors. Cloned purified L. infantum TR was utilized to develop a homogeneous bioluminescent substrate to show a physical signal of its binding with NADP. Before conducting HIT, the stability of TR, NADPH and all other working solutions were tested for their stability. The investigators obtained 290 compounds. However, only 64 were confirmed using dose-response curves for TR inhibition activity with IC50 potencies ranging from 2-35 $\mu \mathrm{M}$. The investigators categorized these compounds into three groups according to their dose-response curves for TR inhibition. Out of 36 compounds that reached $\sim 100 \%$ maximum TR inhibition, the investigators selected compound No. 3 \{[2-(diethyl amino) ethyl 4-[(3-(4-nitrophenyl)-3oxopropyl) amino] benzoate]\} for several reasons: low molecular weight, high solubility, its visual structural inspection, and its relative structural complexity with respect to its analogs.

Using surface plasmon resonance, the investigators evaluated the selected compound for both its binding and kinetics with TR, as well as its competitive activity towards either TS2 or NADPH. The obtained results revealed that compound (3) showed significant fast on/ fast off kinetics with TR, and was competitive for NADPH, without altering TS2. The investigators extended their experiments to investigate the mechanism of inhibition exerted by compound (3) on TR and to identify its binding site using X-ray crystal structure. It was found that compound (3)-TR complex allowed the investigators to identify an unique site (a pocket) on TR that prevents NADPH entrance, and functionally impedes TS2 reduction. Interestingly, this unique site is absent in human homologs (glutathione reductase, hGR), as evident when the investigators performed a similar study on hGR using the selected compound. Finally, efficacy of compound (3) was evaluated as antiproliferative drug against $L$. infantum promastigotes, and it induced $100 \%$ mortality after three days. Compiled from "Identification and binding mode of a novel Leishmania trypanothione reductase inhibitor from high throughput screening." PLoS Negl Trop Dis 2018 Nov 26; 12(11): e0006969. 